\title{
Integrated Dual-Band Antenna System Design Incorporating Cell Phone Bezel
}

\author{
Zhijun Zhang, Senior Member, IEEE, Wenhua Chen, Member, IEEE, Zhenghe Feng, Senior Member, IEEE, and \\ Magdy F. Iskander, Fellow, IEEE
}

\begin{abstract}
This letter describes a method to integrate a cell phone metal bezel in the design of dual-band antenna system. The proposed design includes a hybrid slot and inverted $L$ antennas and covers both the GPS $(1.575 \mathrm{GHz})$ and WLAN $(2.4 \sim$ 4.484 GHZ) frequency bands. A prototype antenna was manufactured on a FR4 board and both S-parameters and radiation patterns are measured, thus validating the simulation results.
\end{abstract}

Index Terms-Cellular radio, multiple frequency antennas.

\section{INTRODUCTION}

I $\mathrm{N}$ THE past few years, more and more cell phone designs use metal bezels as a cosmetic feature on the front panel. Most major phone manufactures currently have some phone models using metal bezels. The metal bezel does have some mechanical advantages besides the cosmetic appeal, including a more resistant to wearing, and it is also stronger than a plated plastic bezel. The use of metal bezels, however, is also associated with liability concerns such as those related to causing ESD when floating bezel is used. For this reason, metal bezels are required to be well grounded and this often interferes with the antenna performance. After grounding the bezel, it becomes equivalent to extending the ground plane out of a traditional IFA or PIFA antenna by $2 \sim 3 \mathrm{~mm}$, which decreases both the antenna efficiency and bandwidth.

In this letter, a new dual-band hybrid antenna design which includes a metal bezel as part of the design is described. This new design not only overcomes the undesirable issues associated with the use of metal bezel, but also uses the metal bezel to enhance the antenna performance. A traditional dual-band IFA or PIFA antenna needs at least an area with a width of $5 \mathrm{~mm}$, which includes both the antenna metal parts and to keep out an area to any nearby shielding or components. The propsed dual-band

Manuscript received April 02, 2008; revised April 20, 2008. First published May 16, 2008; current version published December 30, 2008. This work was supported by the National Basic Research Program of China under Contract 2007CB310605, in part by the National High Technology Research and Development Program of China (863 Program) under Contract 2006AA01Z265, and in part by the National Natural Science Foundation of China under Contract 60496318.

Z. Zhang, W. Chen, and Z. Feng are with the Department of Electronic Engineering, Tsinghua University, Beijing 100084, China (e-mail: zjzh@tsinghua. edu.cn; chenwh@mail.tsinghua.edu.cn; fzh-dee@ mail.tsinghua.edu.cn).

M. F. Iskander is with HCAC, University of Hawaii at Manoa, Honolulu, HI 96822 USA (e-mail: Magdy.iskander@gmail.com; iskander@spectra.eng. hawaii.edu).

Color versions of one or more of the figures in this letter are available online at http://ieeexplore.ieee.org.

Digital Object Identifier 10.1109/LAWP.2008.2000573 antenna takes advantage of the mechanical gap, which already exists between the bezel and PCB board, to fulfill the ground clearance requirement, thus decreases the width of the antenna area to $1 \mathrm{~mm}$.

Hybrid or dual-band antennas are a category of antenna designs which combines two or more different antenna technologies to improve the overall performance and provide multi-band coverage. Ravipatis et al. [1] combined a shorted annular ring and an open-ended circular waveguide to form a dual-band antenna. Esselle et al. [2] reported a hybrid slot and a dielectric resonator antenna (DRA) to achieve multi-band performance. Ding et al. [3] introduced a hybrid antenna with two slots and a DRA to form a tri-band antenna. Lin et al. [4] proposed a hybrid coplanar waveguide (CPW) feed antenna composed of a slot and a printed dipole to generate dual-band resonances.

In cell phone designs, several antennas which cover different bands, need to share a same compact volume. Mutual coupling among antennas often degrades the antennas efficiencies and also makes it difficult to achieve good isolation. It is, therefore, an attractive feature if the individual antennas are integrated into one in the design process to form a multi-band antenna that radiate effectively in each of the desired bands. It should be noted that, not all bands are suitable to be integrated into a single antenna, GSM or UMTS bands have high transmit power and will interference with other bands, thus they have to use exclusive antenna. The Bluetooth and GPS antennas are, however, more appropriate to be combined. A Bluetooth transceiver transmits at relatively lower power, below $4 \mathrm{dBm}$ for class 2 devices, and thus the out-band rejection requirement of a diplexer is reasonable. The GPS and Bluetooth bands are well separated in frequency, so the roll-off requirement is also easy to be achieved. Several readily available diplexers are suitable to separate these two bands inside the RF front end.

The antenna design described in this letter includes a hybrid slot and inverted L structure to cover both GPS $(1.575 \mathrm{GHz})$ and WLAN (2.4 $4.484 \mathrm{GHZ)}$ frequency bands and uses the bezel as integrated part in the design.

\section{DESIGN APPROACH}

The proposed antenna consists of an inverted L element and a slot element. The prototype is made of a $110 \mathrm{~mm} * 60 \mathrm{~mm} 1 \mathrm{~mm}$ thick FR4 PCB $\left(\varepsilon_{\mathrm{r}}=4.5\right)$. Both the silver and orange areas in Fig. 1(c) are made of copper, which simulate bezel and circuit board respectively. The Y-axis in Fig. 1(c) is perpendicular to the plate. As shown in Fig. 1(c), the top part of the bezel has also been used to form the antenna. When the hybrid antenna is radiating in the slot mode at the GPS band, which is shown in Fig. 1(a), the bezel is part of the ground structure which forms 


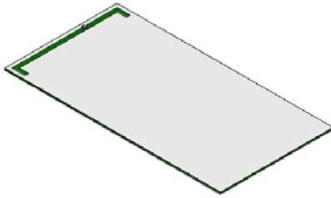

a.

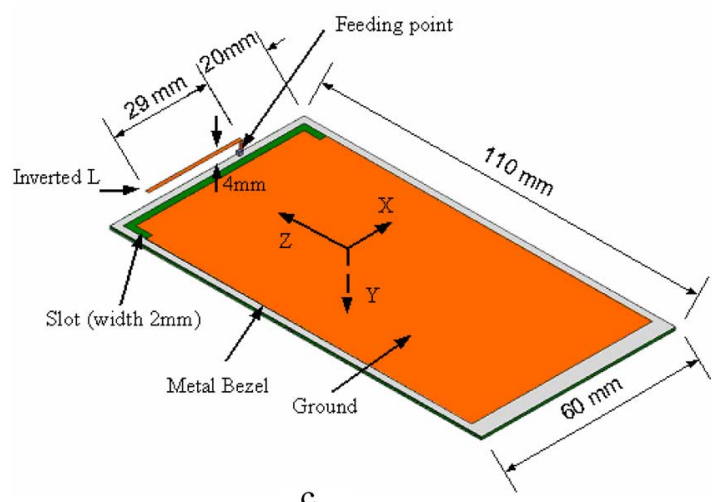

Fig. 1. Mechanical drawing of the proposed antenna. (a) Slot antenna; (b) L antenna; (c) hybrid L and slot antenna.

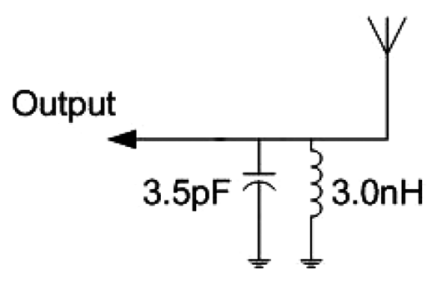

Fig. 2. Matching network used at the feeding point.

the slot. When the antenna is radiating in the L antenna mode at Bluetooth frequency, as shown in Fig. 1(b), the bezel is the ground.

The L element is above the bezel and flushes to the edge of the bezel. The length of the L element is $29 \mathrm{~mm}$ and the clearance between the element and the bezel is $4 \mathrm{~mm}$. The feeding point is located on the end of the L element as shown in Fig. 1(c). The antenna has a single feed. The feed directly excites the L element which radiates at $2.4 \mathrm{GHz}$ and use the L element to parasitically excite the slot which radiates at $1.575 \mathrm{GHz}$. The feeding point is $20 \mathrm{~mm}$ away from the PCB corner. The width of the L element is $1 \mathrm{~mm}$. The width of the slot element is $2 \mathrm{~mm}$, and the total length of the slot is $62 \mathrm{~mm}$. The width of the bezel around the slot is $2 \mathrm{~mm}$. The bezel and the ground are connected everywhere except around the slot. Other dimensions of the radiating elements are shown in Fig. 1(c). A two-component matching network, shown in Fig. 2, includes a $3.5 \mathrm{pF}$ shunt capacitor and a $3.0 \mathrm{nH}$ shunt inductor. This matching network is used at the feeding point to improve the impedance matching of the L element and hence the efficiency of the entire antenna system.

\section{EXPERIMENT RESULT}

Fig. 3 shows the measured return loss, with values of $-19 \mathrm{~dB}$ at $1.575 \mathrm{GHz}$; and $-7.5 \mathrm{~dB}$ and $-10 \mathrm{~dB}$ at $2.4 \mathrm{GHz}$ and $2.482 \mathrm{GHz}$ which are the band edges of the Bluetooth band.

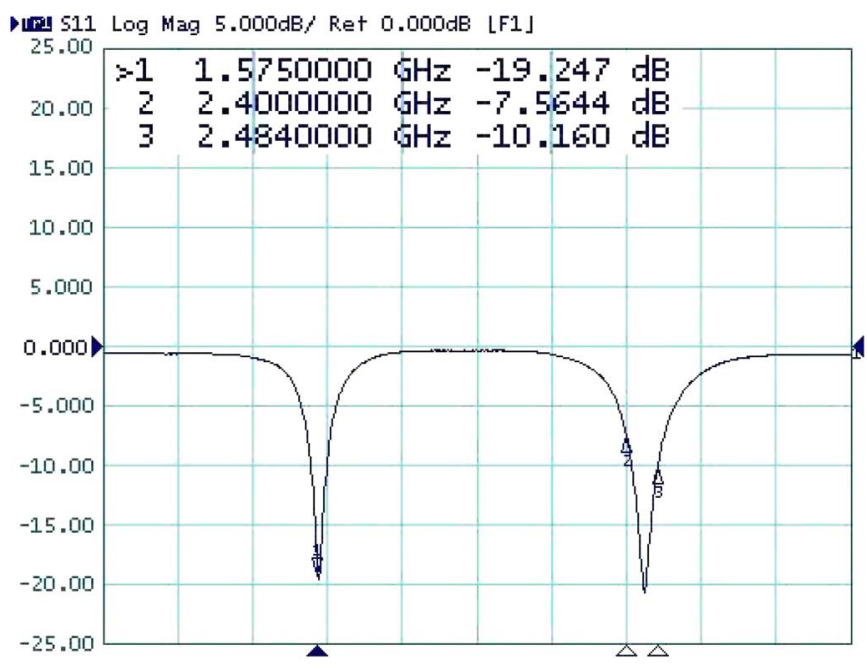

Fig. 3. Measured return loss.

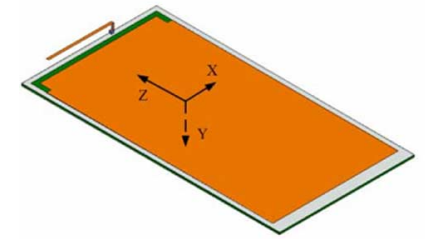

a

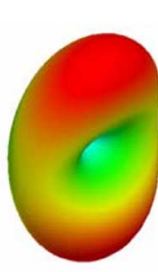

b

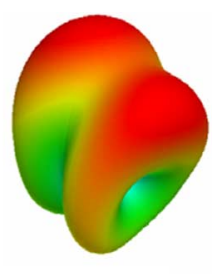

c
Fig. 4. Simulated 3D radiation pattern of different antenna modes. (a) Coordinates; (b) slot antenna only at $1.575 \mathrm{GHz}$; (c) L antenna only at $2.45 \mathrm{GHz}$.

While the resulting bandwidth at the $2.4 \mathrm{GHz}$ meets the requirement of the current project, wider bandwidth could be achieved by increasing the $4 \mathrm{~mm}$ distance between the L element and the bezel.

Fig. 4 shows the simulated 3D radiation patterns of different antenna modes at different frequencies. The Y-axis in Fig. 4(a) is perpendicular to the plate. The Fig. 4(b) shows the 3D pattern of the slot radiation mode, which represents a slot only antenna, as shown in Fig. 1(a), when the antenna is excited at $1.575 \mathrm{GHz}$. Fig. 4(c) shows the result when an L element only antenna, as shown in Fig. 1(b), when the antenna is excited at $2.45 \mathrm{GHz}$.

Fig. 5 shows the measured and simulated radiation patterns, which are normalized to the maximum at 1.575 and $2.44 \mathrm{GHz}$, respectively. Angle 0 corresponds to the $+\mathrm{Z}$ axis shown in Fig. 4(a). Fig. 5(a) shows the patterns of hybrid antenna at $1.575 \mathrm{GHz}$. Comparing with the pure slot antenna results shown in Fig. 4(b), it is clear that the hybrid antenna works in the slot mode at the $1.575 \mathrm{GHz}$. Fig. 5(b) shows the results at $2.45 \mathrm{GHz}$. At this frequency the radiation pattern of the hybrid antenna, which has a slot on the ground plane, is different from the ideal L element antenna results shown in Fig. 4(c), which has a solid ground plane.

\section{CONCLUSION}

The letter describes a hybrid slot and inverted L dual-band antenna structure. In this design the metal bezel which is often used for mechanical and cosmetic purposes is utilized as part 


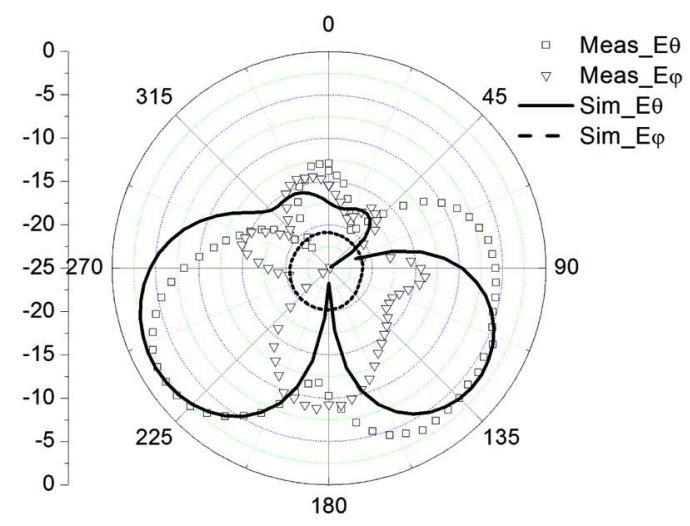

$\mathrm{x}-\mathrm{z}$ plane

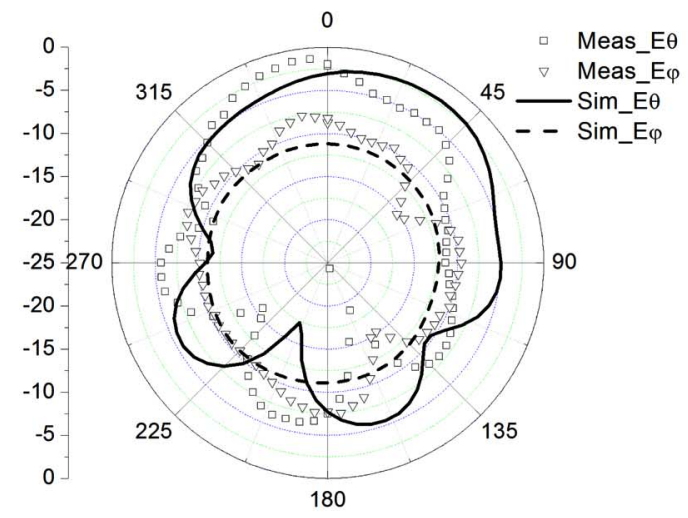

$\mathrm{X}-\mathrm{z}$ plane

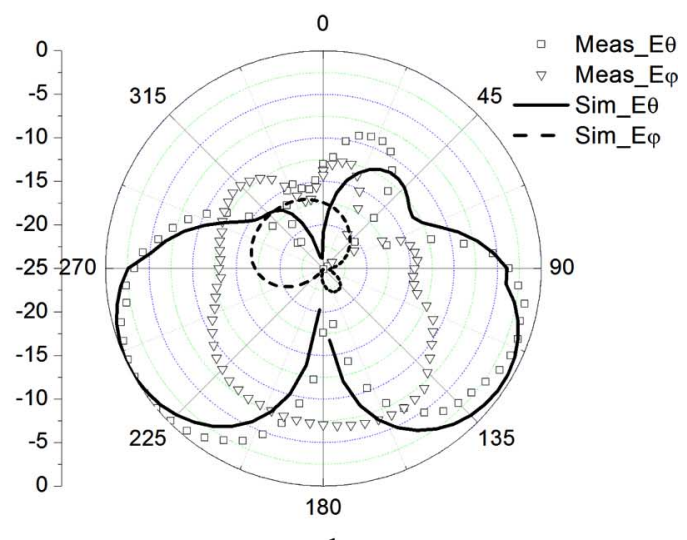

y-z plane

a.

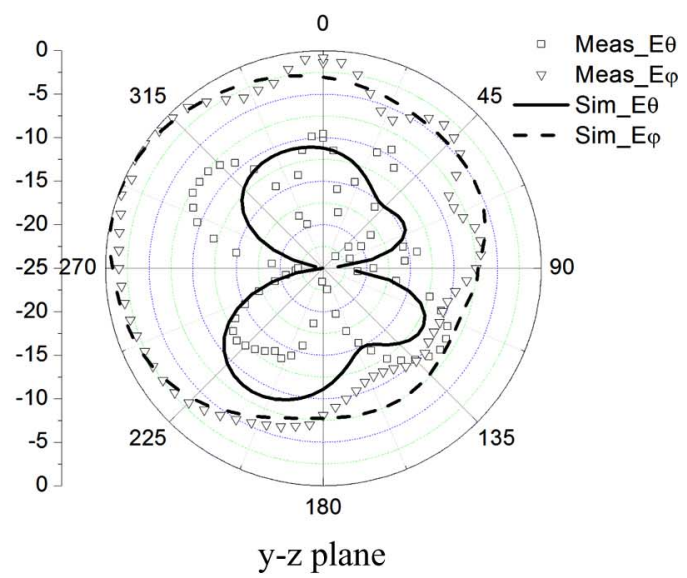

b.

Fig. 5. Measured radiation patterns at both $1.575 \mathrm{GHz}$ and $2.44 \mathrm{GHz}$. (a) $1.575 \mathrm{GHz}$ patterns; (b) $2.44 \mathrm{GHz}$ patterns.

of the radiator. The described design, therefore, not only overcomes some of the concerns regarding ESD issues when using metal bezels, it also integrates the bezel as a useful part of the antenna system. The designed antenna covers two bands which are GPS $(1.575 \mathrm{GHz})$ and WLAN $(2.4 \sim 4.484 \mathrm{GHz})$. The return loss is $-19 \mathrm{~dB}$ at $1.575 \mathrm{GHz}$; and $-7.5 \mathrm{~dB}$ and $-10 \mathrm{~dB}$ at band edges of $2.4 \mathrm{GHz}$ band.

\section{REFERENCES}

[1] C. Ravipatis and A. Zaghloul, "A hybrid antenna element for dual-band application," in Proc. IEEE Antennas Propag. Society Int. Symp., 2004 vol. 4, pp. 4020-4023.

[2] K. Esselle and T. Bird, "A hybrid-resonator antenna: Experimental results," IEEE Trans. Antennas Propag., vol. 53, no. 2, pp. 870-871, 2005.

[3] Y. Ding, K. Leung, and Y. Or, "Dualband DRA-slot hybrid slot antenna," in Proc. IEEE Antennas Propag. Society Int. Symp., 2007, vol. 4, pp. 976-979.

[4] X. Lin and C. Yu, "Dual-band CPW-fed hybrid antenna," Electron. Lett., vol. 43, no. 11, pp. 599-600, 2007. 